\title{
Local delivery of Bone Morphogenetic Protein-2 from near infrared-responsive hydrogels for bone tissue regeneration.
}

Silvia Sanchez-Casanova ${ }^{a, b, \neq}$, Francisco M. Martin-Saavedra ${ }^{a, b, \neq, *}$, Clara EscuderoDuch $^{a, b}$, Maria I. Falguera Uceda ${ }^{a}$, Martin Prieto ${ }^{c, b, d}$, Manuel Arruebo ${ }^{c, b, d}$, Paloma Acebo $^{e}$, Mario Fabiilli ${ }^{f, g, h}$, Renny T. Franceschi ${ }^{i, h, j}$, Jesus Santamaria ${ }^{c, b, d}$, Nuria Vilaboa $^{a, b, *}$

aHospital Universitario La Paz-IdiPAZ, Paseo de la Castellana 261, Madrid, 28046 Spain.

${ }^{\mathrm{b} C I B E R}$ de Bioingenieria, Biomateriales y Nanomedicina, CIBER-BBN, Spain

'Departmento de Ingenieria Quimica, Instituto de Nanociencia de Aragon (INA), Universidad de Zaragoza, Campus Rio Ebro. Edificio I+D. C/ Mariano Esquillor s/n, Zaragoza, 50018 Spain

${ }^{\mathrm{d} I n s t i t u t o ~ d e ~ C i e n c i a ~ d e ~ M a t e r i a l e s ~ d e ~ A r a g o n, ~ C o n s e j o ~ S u p e r i o r ~ d e ~ I n v e s t i g a c i o n e s ~ C i e n t i f i c a s ~}$ (ICMA-CSIC), Universidad de Zaragoza, Zaragoza, 50009, Spain

eUnidad Funcional de Investigación de Enfermedades Crónicas (UFIEC), Instituto de Salud Carlos III, Crta. Majadahonda a Pozuelo km.2, Majadahonda, Madrid 28220, Spain

${ }^{\mathrm{f}}$ Applied Physics Program, University of Michigan, Ann Arbor, MI USA

gDepartment of Radiology, University of Michigan Health System, Ann Arbor, MI USA

hDepartment of Biomedical Engineering, University of Michigan, Ann Arbor, MI USA

iDepartment of Periodontics and Oral Medicine, School of Dentistry, University of Michigan, Ann Arbor, MI USA

jDepartment of Biological Chemistry, University of Michigan Medical School, Ann Arbor, MI USA

‡ These authors contributed equally

\section{Introduction}

Much of biomedical research in the field of mineralized tissue engineering is currently devoted to developing technologies that enhance the healing of injured bone. In order to orchestrate bone regeneration, some therapeutic strategies have focused on the controlled delivery of morphogens, often in the form of proteinaceous growth factors, a 
challenging endeavour. Given their central role in bone development, remodeling and regeneration [1], considerable emphasis has been placed on the development of methods for delivering bone morphogenetic proteins (BMPs) to bone regeneration sites. BMP-2, involved in committing multipotent stem cells towards the osteogenic lineage [2], is also capable of increasing the migration of endogenous progenitors to defect sites [3] and has strong osteoinductive activity both in vitro and in vivo [4]. As a therapeutic resource, recombinant growth factors are expensive and cumbersome to manufacture because eukaryotic expression systems are needed in order to ensure the correct protein folding and post-translational modifications required for product safety and efficacy. Added to this disadvantage is the fact that many recombinant growth factors possess short half-lives, e.g. systemically administered BMP-2 has a half-life of only $7 \mathrm{~min}$ as shown in animal studies including non-human primates [5, 6]. For this reason, supraphysiological doses need to be administered in order to reach and sustain a therapeutically-effective dose. However, use of high dose BMPs increases the risk of side effects. Typically, recombinant BMP-2 is administered at doses in the milligram range, exceeding by a million times the amounts of the endogenous growth factor. Thus, the supraphysiological BMP-2 doses administered locally during surgical procedures have been linked with serious complications, such generalized hematomas in soft tissue [7], exaggerated inflammatory response in proximal humeral fractures [8], unicameral bone cysts [9] and infections in open tibial fractures [10]. In spinal fusions, reported adverse effects include seroma, swelling, ectopic/heterotopic bone formation, vertebral osteolysis, retrograde ejaculation, radiculitis, dysphagia, graft subsidence and postoperative nerve root compression [11-18]. A controversy also exists concerning postoperative cancer risk as the incidence rate of new cancers two years after spinal arthrodesis was higher in patients receiving high doses of BMP-2 [19]. 
Gene therapy is an alternative technology for delivering gene products in situ. Transgene expression in host cells is able to produce therapeutic proteins that undergo proper post-translational modifications and folding. Bone healing after BMP-2 gene transfer has proven to be feasible. Upon direct percutaneous injection in rats, adenoviral constructs encoding BMP-2 healed femoral defects to an extent dependent on the dose of the administered vector [20, 21]. When delivering autologous or allogenic bone marrow stem cells (BMSC) transduced with adenoviral vector carrying a BMP-2 gene to rat femoral defects, efficient bone healing was also detected [22, 23]. In larger animals such rabbits or sheep, local administration of adenovirally-encoded BMP-2 healed femur segmental defects [24, 25]. The combination of tissue engineering and gene therapy approaches has also proven effective, as shown in tibial defects of goats implanted with scaffolds composed of biphasic calcined bone containing autologous BMSC transduced with BMP-2 [26]. Most BMP-2 gene expression systems employ unregulated promoters, which may produce excessive amounts of recombinant protein leading to side effects similar to those observed after recombinant BMP-2 protein delivery. Currently available pharmacologically and/or physically activated expression systems can provide tight control over the level, duration and spatial localization of one or more transgenic morphogens, thus optimizing the safety and effectiveness of gene therapy [27]. Our previous work developed regulatory gene switches that combine the promoter of the highly heat-inducible HSP70B gene (HSPA7) and a small moleculedependent transactivator and allow deliberate control over spatial as well as temporal transgene expression [28]. A particular heat-activated and dimerizer-dependent switch utilizes the activated $H S P 70 B$ promoter to drive the expression of a chimeric transactivator that in the presence of rapamycin or its non-immunosuppressive analogs acquires transcriptional competence and controls the expression of a therapeutic 
transgene [29]. Using focused ultrasound as a heating source for cell constructs, this gene switch controlled in vitro both the timing and spatial distribution of BMP-2 [30] as well as the localized in vivo expression of a firefly luciferase reporter [30, 31].When the expression of human vascular endothelial growth factor 165 (hVEGF 165$)$ was placed under the control of the gene switch, we could define precise spatiotemporal patterns of VEGF production in vivo using NIR irradiation of photothermal cell constructs [32]. NIR light with wavelength range from 650 to $900 \mathrm{~nm}$ is minimally absorbed by skin and underlying tissues $[33,34]$ and can stimulate photoabsorbers embedded in the depth of a tissue to transduce incident energy into local heating. In the present work, we triggered regulated BMP-2 expression in cells harbouring a heat-activated and rapamycindependent gene switch that were loaded in NIR-responsive scaffolds based on fibrin and plasmonic gold nanoparticles. To investigate the bone healing capacity of this gene therapy approach we used the photothermal constructs to fill a critical-size calvarial defect created in immunocompetent mice. Administration of dimerizer followed by NIR irradiation of the implant induced BMP-2 production, which resulted in the formation of new bone.

\section{Materials and methods}

\subsection{NIR-responsive nanoparticles}

Hollow gold nanoparticles (HGNP) were synthesized following a scaled up variation of a previously reported method [35]. Briefly, $800 \mu \mathrm{L}$ of $0.4 \mathrm{M}$ cobalt chloride hexahydrate and $1.6 \mathrm{~mL}$ of $0.1 \mathrm{M}$ sodium citrate tribasic dihydrate were mixed with 400 $\mathrm{mL}$ of deionized water in a round-bottom flask. The solution was de-aired by bubbling with argon for $45 \mathrm{~min}$. Then, $4 \mathrm{~mL}$ of a $1 \mathrm{wt}$ \% solution of poly(vinylpyrrolidone) (PVP; MW $=55 \mathrm{KDa}$ ) and $900 \mu \mathrm{L}$ of $0.1 \mathrm{M}$ sodium borohydride were injected under 
magnetic stirring. The solution turned from pale pink to dark brown after a few seconds, indicating the formation of cobalt NP. To complete the hydrolysis of sodium borohydride, the NP dispersion was maintained under magnetic stirring and argon bubbling for additional $15 \mathrm{~min}$. Next, $380 \mathrm{~mL}$ of the dispersion were transferred to a beaker that contained $100 \mathrm{~mL}$ of $0.38 \mathrm{mM} \mathrm{HAuCl}_{4}$ and were kept under magnetic stirring for $30 \mathrm{~min}$ to allow the galvanic replacement of cobalt by gold. Finally, obtained HGNP were purified by several centrifugal washings with deionized water at $13,000 \mathrm{~g}$ for $10 \mathrm{~min}$ and stored as lyophilized powder at $-20{ }^{\circ} \mathrm{C}$. Freeze dried HGNP were resuspended in sterile deionized water at a final concentration of $1 \mathrm{mg} \mathrm{mL}^{-1}$. To reduce aggregation, HGNP dispersions were sonicated for $10 \mathrm{~min}$ in an ultrasonic bath (Bransonic, Emerson) operating at $40 \mathrm{kHz}$ and $80 \mathrm{~W}$ of output power. The average diameter of HGNP was $44 \pm 9.34 \mathrm{~nm}$ (size range was 10-160 nm, $96 \%$ were below 90 $\mathrm{nm}$ ), as determined by quantitative analysis of transmission electron microscopy (TEM) images obtained using a field emission gun microscope (FEI Tecnai T20) operated in the bright field mode and at an accelerating voltage of $200 \mathrm{keV}$. Gold content of HGNP was $59.02 \pm 0.43 \mathrm{wt}$ \% $\%$ as determined by atomic emission spectroscopy analysis using a microwave plasma-atomic emission spectrometer (MP-AES model 4100, Agilent technologies). An extensive physicochemical characterization of HGNP can be found in our previous works[36,37].

\subsection{Vector construction}

The cDNA sequence of (RAT)Bmp2 gene (NM_017178.1) was PCR-amplified from pBluescipt-SK-BMP-2rat [38] using primers 5'GAATTGGGTACCGGGTTTAAACTCGAG-3’ and 5’CTGCAGGGATCCGGCACGAG-3’. The PCR product was digested with BamHI and PmeI and then ligated to BamHI/PmeI-digested pGene/V5-His A (Invitrogen) to obtain 
pGeneRatBMP. A version of this plasmid, which contains a GAL4 promoter controlling Bmp2 coding sequence as well as adjacent bovine growth hormone poly(A) sequences flanked by ClaI sites, was prepared using the Quikchange site-direct mutagenesis kit (Agilent) with the following oligonucleotides and their complements: 5'GGATCGGGAGATCGTACCTATCGATTACGCGGGTCGAAGCGG-3' and 5'GGATGCGGTGGGCTCTATGGCTATCGATGCGGAAAGAACCAG-3'. The resulting construct pGeneRatBMP-ClaIx2 served as template for PCR amplification using primers 5'-GAGATCGTACCTAACGCGTACGC-3' and 5'CTGGTTCTTTCCGCATCGATAGCC-3’. The PCR product was digested with MluI and ClaI and ligated to the larger fragment of MluI/ClaI-digested pLH-Z ${ }_{12} \mathrm{I}-\mathrm{PL}$ (ARIAD Gene Therapeutics Inc). The resulting plasmid pLH-GAL4-ratBMP2-BGHpA was digested with BamHI and ClaI to obtain a fragment containing the BMP-2-encoding sequence and the adjacent $\mathrm{BGH}$ poly(A) sequence. The fragment was ligated to a BamHI/ClaI/-digested version of pLH-Z ${ }_{12} \mathrm{I}-\mathrm{PL}$ in which a BamHI site was inserted at the multicloning site of pLH-Z ${ }_{12} \mathrm{I}-\mathrm{PL}$ using the QuikChange method and oligonucleotide 5'-TTGCCCTGCAGCGGGGATCCCACTAGTCGAGATC and its complement. Resulting plasmid was named pLH-Z ${ }_{12} \mathrm{I}-\mathrm{PL}-\mathrm{ratBMP}-2$. All subcloning and mutagenesis steps were monitored by restriction analysis and nucleotide sequencing.

\subsection{Cell culture and isolation of cell lines}

Mouse embryo fibroblast C3H/10T1/2 cells (clone 8, ATCC CCL-226) and derived cell lines were cultured in growth medium composed by Eagle's minimal essential medium (EMEM) supplemented with $10 \%(\mathrm{v} / \mathrm{v})$ fetal bovine serum (FBS), $100 \mathrm{U} \mathrm{mL}^{-1}$ penicillin, and $0.1 \mathrm{mg} \mathrm{mL}^{-1}$ streptomycin. A C3H/10T1/2 clone, stably harbouring the cDNAs of the two modules of a chimeric dimerizer-dependent transactivator under the control of a promoter comprising components of the highly heat-inducible HSP70B 
gene and the activated transactivator-responsive promoter [29], was transfected with pLH-Z ${ }_{12} \mathrm{I}-\mathrm{PL}-\mathrm{ratBMP}-2$ and stable transfectants were selected using $600 \mu \mathrm{g} \mathrm{mL}{ }^{-1}$ hygromycin B and $1200 \mu \mathrm{g} \mathrm{mL} \mathrm{m}^{-1}$ G418 (InvivoGen). Single-cell clones were screened for BMP-2 secretion after treatment with $10 \mathrm{nM}$ rapamycin and heating at $44{ }^{\circ} \mathrm{C}$ for 30 min in a waterbath. A highly inducible cell line that exhibited negligible levels of BMP2 secretion after heat treatment or rapamycin alone was isolated and termed C3H-BMP$2^{\text {high }}$. C3H/10T1/2-fLuc cells, derived from C3H/10T1/2, clone 8, cells as described elsewhere [4], stably harbour a heat-activated and dimerizer-dependent gene switch that controls a firefly luciferase ( $f \mathrm{Luc}$ ) gene. Cells were cultured in a humidified $5 \% \mathrm{CO}_{2}$ atmosphere at $37{ }^{\circ} \mathrm{C}\left(37^{\circ} \mathrm{C} / 5 \% \mathrm{CO}_{2}\right)$. Clonal cells were maintained under continous selection with $1200 \mu \mathrm{g} \mathrm{mL} L^{-1} \mathrm{G} 418$ and $600 \mu \mathrm{g} \mathrm{mL}{ }^{-1}$ hygromycin B.

\subsection{Preparation and induction of NIR-responsive hydrogels}

Bovine fibrinogen (fbg; Merck) was dissolved in ice-cold EMEM at a concentration of $40 \mathrm{mg} \mathrm{mL}-1$ of clottable protein. Freeze dried HGNP were suspended in sterile deionized water at a final concentration of $1 \mathrm{mg} \mathrm{mL}^{-1}$, sonicated for $10 \mathrm{~min}$ in an ultrasonic bath (Bransonic, Emerson) operating at $40 \mathrm{kHz}$ and $80 \mathrm{~W}$ of output power, and then added to the fbg solution at $20-40 \mu \mathrm{g} \mathrm{mL} \mathrm{m}^{-1}$. Next, 0.8 volumes of EMEM containing $4 \times 10^{6} \mathrm{C} 3 \mathrm{H} / 10 \mathrm{~T} 1 / 2-$ fLuc or C3H-BMP-2 ${ }^{\text {high }}$ cells $\mathrm{mL}^{-1}$ or not containing cells were added to the mixture. To catalyze fbg polymerization, 0.2 volumes of an icecold solution of $20 \mathrm{U} \mathrm{mL}^{-1}$ bovine thrombin (Merck) in EMEM were added. After mixture homogenization by pipetting, $0.25 \mathrm{~mL}$ of suspension were distributed per well in 48-well tissue culture plates. Suspensions were allowed to clot for $30 \mathrm{~min}$ at $37{ }^{\circ} \mathrm{C} / 5$ $\% \mathrm{CO}_{2}$. After clotting, 1 hydrogel volume of EMEM containing $20 \%$ FBS was added to the construct which was then further incubated at $37{ }^{\circ} \mathrm{C} / 5 \% \mathrm{CO}_{2}$ for $4 \mathrm{~h}$ to equilibrate serum content. Finally, hydrogel medium was replaced with 1 hydrogel volume of fresh 
growth medium. Hydrogels harbouring C3H/10T1/2-fLuc and C3H-BMP-2 ${ }^{\text {high }}$ cells were named NIR-fLuc-HG and NIR-BMP2-HG, respectively. In some experiments, hydrogels were prepared as above described, but lacked HGNP.

The NIR irradiation set-up consisted of an $808 \mathrm{~nm}$ laser module with $400 \mu \mathrm{m}$ fiber coupling (MXL-III model; Changchun New Industries Optoelectronics Technology Co., Ltd.) that was connected to a fixed focus collimator $(f=11.07 \mathrm{~mm}, \mathrm{NA}=0.26$; Thorlabs). The laser diode was driven by a variable power supply unit (PSU-III-LED model, Changchun New Industries Optoelectronics Technology Co., Ltd.). Output power was measured using a silicon photodiode sensor (Model PD300-3W, Ophir Laser Measurement Group). The optical fiber was coupled to a micro positioning system. For in vitro assays, hydrogels were immersed in $0.5 \mathrm{~mL}$ of fresh growth media with or without the indicated doses of dimerizer. After $60 \mathrm{~min}$ of incubation at $37^{\circ} \mathrm{C} / 5 \% \mathrm{CO}_{2}$, the hydrogels were irradiated with the collimated NIR laser inside a thermostaticallycontrolled chamber (Model Stuart SI60D, Fisher Scientific Afora) to establish an environmental temperature of $37^{\circ} \mathrm{C}$. Hydrogels that did not contain HGNP, or that were not NIR-irradiated, were employed as controls. One day after irradiation, media were collected and BMP-2 levels were measured in the conditioned media using a commercially available ELISA kit (R\&D Systems). The experimental time schemes used for NIR irradiation of hydrogels are shown in Figs. 1C, 2A, 3C and S3A. Temperature changes in the hydrogels or implantation areas were monitored by infrared (IR) thermography using a Testo 875-2i thermal imaging camera (Testo, Inc.).

\subsection{Micromass culture differentiation assays}

$\mathrm{C} 3 \mathrm{H} / 10 \mathrm{~T} 1 / 2$, clone 8 , cells on passage 3-5 were suspended at $10^{7}$ cells $\mathrm{mL}^{-1}$ in growth medium. Next, $10 \mu \mathrm{L}$ of the cell suspension were applied to the center of the well of a 
12-well tissue culture plate. Cells were incubated at $37^{\circ} \mathrm{C} / 5 \% \mathrm{CO}_{2}$ for $2 \mathrm{~h}$ and then 1 $\mathrm{mL}$ of growth medium was slowly added to flood cell micromass. After $24 \mathrm{~h}$ of incubation at $37^{\circ} \mathrm{C} / 5 \% \mathrm{CO}_{2}$, medium was removed and cell cultures were treated with 1-100 ng $\mathrm{mL}^{-1}$ of recombinant human BMP-2 (rBMP-2; R\&D) and/or 10-100 nM dimerizer. After 5 days of incubation, media were replaced with fresh media containing 1-100 ng mL $\mathrm{m}^{-1} \mathrm{rBMP}-2$ and/or 10-100 nM dimerizer and incubated for further 5 days. In some experiments, micromasses were co-cultured with NIR-responsive hydrogels that were placed in the upper chamber of a transwell system (Corning). Right after NIR irradiation in a final volume of $0.5 \mathrm{~mL}$ of growth medium, the hydrogels were placed in the upper chamber of the transwell system and cultured for 5 days in the presence or absence of dimerizer in a final volume of $1 \mathrm{~mL}$ of growth medium. Then, hydrogels were removed from the co-culture system, irradiated as before in the presence or absence of dimerizer and placed again in the upper chamber of the transwell system for additional 5 days in the presence or absence of dimerizer. At the end of the incubation periods, micromasses were fixed with $4 \%$ paraformaldehyde in phosphate-buffered saline (PBS) for 15 min and then incubated overnight at $37^{\circ} \mathrm{C}$ with $0.05 \%$ alcian blue (Merck) dissolved in 75 \% EtOH:0.1 M HCl (4:1). After intensive washing with $0.1 \mathrm{M}$ $\mathrm{HCl}$, micromasses were scanned using a document scanner (ScanJet 3400C, Hewlett Packard) and visualized using Olympus BX41 microscope (Olympus). The experimental time schemes used for assays with micromasses are shown in Figs. 3A and 3B.

\subsection{Cell viability assays}

Viability of cells entrapped in hydrogels was investigated using the alamarBlue-based assay. After washing with PBS, samples were incubated for $4 \mathrm{~h}$ at $37^{\circ} \mathrm{C} / 5 \% \mathrm{CO}_{2}$ in growth medium containing $10 \%$ alamarBlue dye (Biosource). After excitation at 530 
$\mathrm{nm}$, emitted fluorescence at $590 \mathrm{~nm}$ was quantified using a Synergy4 Multimode Plate Reader (Biotek). Cell viability was additionally assessed by staining with acetoxymethyl ester of calcein (calcein-AM), which stains esterase-positive cells and ethidium homodimer-1 (EthD-1), a membrane-impermeable fluorescent dye which binds to DNA. Hydrogels were washed with PBS and incubated for $2 \mathrm{~h}$ at room temperature (RT) in cell culture medium containing $2 \mu \mathrm{M}$ calcein-AM and $4 \mu \mathrm{M}$ EthD1 (both from Invitrogen) for viable and damaged cell staining, respectively. The samples were rinsed gently with PBS before examination using a spectral confocal microscope Leica TCS SPE (Leica Microsystems). To visualize cells stained with calcein-AM and EthD-1, samples were excited with $488 \mathrm{~nm}$ and 532 laser lines and emission was collected in 517 and $617 \mathrm{~nm}$ bandwidths, respectively. Images of $4.7 \mathrm{~mm}^{2}$ of the central area of each hydrogel, including the NIR-illuminated region, were acquired. Stacks of 2 $\mu \mathrm{m}$ optical sections were recorded and analyzed using Leica software LAS AF, version 2.0.1, build 2043. Quantification of alive cells was performed using analysis software (Image J/Fiji) [39].

\subsection{Treatment with dimerizers}

For in vitro experiments, rapamycin (Invivogen) was dissolved in N, Ndimethylacetamide (DMA) and the rapalog AP21967 (Clontech) in $100 \%$ ethanol. Rapamycin was used at a final concentration of $10 \mathrm{nM}$ while AP21967 was used at 10$100 \mathrm{nM}$. For in vivo treatments, rapamycin was dissolved in DMA to prepare a stock solution (5 mg mL $\mathrm{m}^{-1}$ ) which was then diluted in a mixture of $50 \%$ DMA, $45 \%$ polyoxyethylene glycol (average molecular weight of $400 \mathrm{Da}$ ) and $5 \%$ polyoxyethylene sorbitan monooleate (all from Merck). A dose of $1 \mathrm{mg} \mathrm{kg}^{-1}$ body weight was injected intraperitoneally in a final volume of $100 \mu \mathrm{L}$. 


\subsection{Microarray analysis of differential gene expression}

Total RNA was extracted using the Animal Tissue RNA Purification Kit (Norgen Biotek Corporation), processed using GeneChip ${ }^{\circledR}$ WT PLUS Reagent kits and hybridized with a GeneChip Mouse Clariom ${ }^{\mathrm{TM}}$ S Array (all from Applied Biosystems). The array, which measures the transcript levels of 22,100 well-annotated genes, was scanned using a GeneChip scanner 3000 7G (Applied Biosystems). Raw data were normalized and gene levels analyzed using the RMA algorithm (Transcriptome Analysis Console Software). For each experimental condition, three RNA replicates corresponding to independent experiments were processed and analyzed. Fold changes between experimental conditions were calculated as ratios of means of gene expression signals. Genes with $\geq 1.500$ - or $\leq 0.666$-fold changes were included for further analysis. Gene ontology analyses were performed using the Database for Annotation, Visualization and Integrated Discovery (DAVID; http://david.abcc.ncifcrf.gov) [40]. Conclusions drawn from microarray experiments were corroborated by reverse transcription and quantitative polymerase chain reaction analysis (RT-qPCR) for a representative number of genes (Table S1). cDNA was prepared from total RNA using the High-Capacity RNA-to-cDNA ${ }^{\mathrm{TM}}$ Kit (Life Technologies). qPCR was performed using TaqMan Gene Expression Assays (Life Technologies, see Table S1 for Assay IDs) and TaqMan Gene Expression Master Mix.

\subsection{Animal experiments}

All procedures were approved by the Animal Welfare Committee of Hospital Universitario La Paz-IdiPAZ and were in compliance with the European Union Directive 2010/63/EU for animal experiments. For critical-size calvarial defects, 5 weeks old C3H/HeNRj mice (Janvier-Labs) were anesthetized with isoflurane (5 \% for induction and $2 \%$ for maintenance; Baxter) and hair was removed from the scalp by 
shaving and depilatory treatment. A coronal incision of $3 \mathrm{~cm}$ was made in the scalp at the occipital level to expose the cranial vault. A $4 \mathrm{~mm}$ diameter defect was created using a tungsten-carbide dental drill (012 gauge; Hager \& Meisinger) attached to a high torque micromotor (Mestra) under continuous irrigation with normal saline (Fresenius). Soft tissues were repositioned and the coronal incision was closed using resorbable suture based on 6-0 coated Vicryl (Ethicon). Bone defect area was filled with $250 \mu \mathrm{L}$ of soluble hydrogel components and mice were kept stationary for 5 min during the in situ polymerization of the implant. For transgene induction assays, rapamycin was administered one and eight days after hydrogel injection. Ninety min after rapamycin administration, mice eyes were covered with aluminum foil to prevent retinal damage and implants were NIR-irradiated for $10 \mathrm{~min}$ at a single spot perpendicular to the bone defect. Temperature changes in the body surface were monitored by IR thermography. To quantify BMP-2 production in implants after NIR irradiation, mice were euthanized and implants were weighed on a precision scale, excised and homogenized in lysis buffer containing 10 mM Tris-Cl (pH 8.0), 1 mM EDTA, 1 \% Triton X-100, $0.1 \%$ sodium deoxycholate, $0.1 \%$ SDS, $140 \mathrm{mM} \mathrm{NaCl}$ and $1 \mathrm{mM}$ PMSF (all from Merck). Extracts were clarified by centrifuging at $16,000 \mathrm{x}$ g for $10 \mathrm{~min}$ at $4{ }^{\circ} \mathrm{C}$ and tested using a commercially available ELISA kit for BMP-2 (R\&D Systems). The experimental time schemes used for in vivo assays are shown in Figs. 4, 5 and S4A.

\subsection{In vivo imaging}

For bioluminescence assays, $50 \mu \mathrm{L}$ of a solution of $40 \mathrm{mg} \mathrm{mL}^{-1}$ D-luciferin (PerkinElmer) in PBS were injected near the implant region and animals were then imaged. Luminescence signal was acquired on a charge-coupled device camera system (IVIS Spectrum, PerkinElmer). Data collected by the IVIS charge-coupled device camera system were analyzed using the Living Image 4.0 software (PerkinElmer). X- 
ray captions were obtained in anesthetized animals using a micro focus tube powered for $34 \mathrm{~s}$ at a voltage of $35 \mathrm{kV}$ and a current of $100 \mu \mathrm{A}$ (IVIS Lumina XR III, PerkinElmer).

\subsection{Histology}

Animals were euthanized and the heads were surgically removed. Samples were fixed with formalin for 4 days and then exposed to a decalcifying solution containing EDTA (Osteodec, Bio-Optica) for 3 days at $4{ }^{\circ} \mathrm{C}$ with daily solution changes. Heads were reconditioned with 3 changes of PBS, 20 min each, and then dissected to remove the nose and lower jaw. Next, samples were dehydrated through a series of graded ethanols, xylol and finally xylene and then embedded in paraffin. After dewaxing and rehydration, seven-micron sections of retrieved samples were stained with Masson's trichrome (Merck).

Hydrogel samples were fixed, dehydrated and embedded in paraffin. After dewaxing and rehydration, five-micron sections were incubated in $0.01 \mathrm{M}$ citrate buffer ( $\mathrm{pH}$ 6.0) and antigen retrieval was performed at $95{ }^{\circ} \mathrm{C}$ for $20 \mathrm{~min}$. Endogenous peroxidase activity was blocked with methanol containing $2.88 \% \mathrm{H}_{2} \mathrm{O}_{2}$ for 10 min at RT. Samples were washed with PBS and then incubated with $1.5 \%$ bovine serum albumin (Merck), $10 \%$ goat serum (Bio-Rad) and $0.05 \%$ Tween 20 (Merck). Samples were incubated overnight at $4{ }^{\circ} \mathrm{C}$ with anti-BMP-2 mouse monoclonal antibody (Abcam) diluted at 1:250. After washing with PBS, samples were incubated for 60 min at RT with antimouse horseradish peroxidase-conjugated polyclonal antibody (Millipore) diluted at 1:200. Next, samples were stained for 7 min at RT with 3,3'-diaminobenzidine (CINtec Histology). 
For imaging of histological sections, an Olympus BX41 microscope (Olympus) was used. For measurements, 6 histological sections from independent regions of the implantation area $(n=5)$ were employed. To assess bone healing in the implanted critical-size bone defect, a scoring method for the qualitative measurement of newly formed bone was used [41]. The defect area, from one edge of the osteotomy to the other, was divided into 135 squares of $50 \mu \mathrm{m}$ each, with a length of 27 squares and a width of 5 squares. To evaluate the degree of bone healing in each square, we applied a scoring system ranging from 0 to 4 . Score 0 corresponded to squares without tissue, score 1 to squares filled only with fibrous tissue, scores 2 and 3 corresponded to squares containing fibrous tissue and a content of mineralized tissue lower and higher, respectively, than $50 \%$, and the score 4 corresponded to areas filled by mineralized tissue. The average value of the scores corresponding to the 135 squares was determined for each analyzed defect. Fiji software [39] was used for the segmentation of the histological images in the histomorphometry analysis and for the quantification of BMP-2 expressing cells.

\subsection{Statistics}

Data are presented as means + standard deviation (SD) of the mean of at least three independent experiments. Statistical analyses were performed by one-way ANOVA followed by Dunnett's multiple comparisons test (Figs. 1, 3 and 4) or two-tailed t-test (Figs. 5, S2, and S3), using GraphPad Prism Version 7.00. Statistical tests of differential gene expression assessed by microarray analysis were performed using the e-bayes limma software included in Transcriptome Analysis Console (Applied Biosystems). The criterion for significance in statistical analysis was set at a $p<0.05$.

\section{Results}




\subsection{NIR-responsive hydrogels for the controlled production of BMP-2}

C3H-BMP-2 ${ }^{\text {high }}$ cells, harbouring a heat-activated and rapamycin-dependent gene switch engineered to control the expression of BMP-2 (Fig. S1), were entrapped within fibrin hydrogels polymerized in situ with 20-40 $\mu \mathrm{g} \mathrm{mL}{ }^{-1}$ HGNP. Irradiation of the resulting constructs NIR-BMP-2-HG with an NIR laser emitting at $44 \mathrm{~mW} \mathrm{~mm}{ }^{-2}$ for 15 min resulted in the generation of local heating. Temperature of hydrogels rapidly increased from $30.17 \pm 0.84{ }^{\circ} \mathrm{C}$ at the time that irradiation started. The increase was proportional to the amount of HGNP used during hydrogel polymerization (Fig. 1A). During NIR illumination, the irradiated surface of NIR-BMP-2-HG polymerized with $30 \mu \mathrm{g} \mathrm{mL} \mathrm{m}^{-1} \mathrm{HGNP}$ experienced a maximum average increase of $11.2 \pm 0.6{ }^{\circ} \mathrm{C}$. As we previously reported, such a moderate photothermal conversion highly induced in vitro and in vivo the expression of a reporter transgene controlled by the heat-activated and dimerizer-dependent gene switch [32]. Consistent with these findings, cells cultured for 1 day in NIR-BMP-2-HG that polymerized with $30 \mu \mathrm{g} \mathrm{mL}{ }^{-1}$ HGNP strongly increased BMP-2 secretion after NIR irradiation in the presence of $10 \mathrm{nM}$ rapamycin (Fig. 1B). NIR irradiation or rapamycin treatment alone did not significantly affect BMP-2 secretion. Next, we investigated whether the BMP-2 levels achieved were dependent on the exposure time to NIR light. As compared to non-irradiated constructs, BMP-2 secretion increased 8 and 20 times after irradiation for 7.5 and $10 \mathrm{~min}$, respectively (Fig. 1C). Immunostaining revealed that the latter exposure time induces the production of BMP-2 in $90 \%$ of the cells included in the hydrogels (Fig. S2). Constructs irradiated for 12.5 and 15 min released lower amounts of BMP-2 than constructs irradiated for 10 min. One day after NIR irradiation, conditioned media were replaced with media lacking rapamycin and constructs were cultured further for three days. At this time, there were no significant differences between the BMP-2 levels in the conditioned 
media of irradiated and non-irradiated hydrogels. A second round of NIR irradiation in the presence of rapamycin, applied five days after the first round, was able to reinduce the release of BMP-2 to the conditioned media of hydrogels. As observed after the first irradiation round, the dynamics of BMP-2 production were dependent on the exposure time to the laser. As compared with non-irradiated NIR-BMP-2-HG, constructs irradiated for 10 or 12.5 min increased BMP-2 secretion about 12 times, while 7.5 or 15 min of light exposure resulted in an 8-fold increase of the cytokine production. The activating treatment affected the fate of the cells included in the constructs, as observed in cell viability analyses performed immediately prior to the second round of NIR irradiation and five days after (Fig. 1D). Cell viability as measured using an alamarBlue-based assay gradually decreased with increasing levels of delivered light energy, likely due to lethal heating at area of laser incidence. In fact, incubation of hydrogels with calcein-AM and EthD-1 disclosed that the percentage of viable cells in the NIR-illuminated region decreased as a function of irradiation time. Interestingly, although prolonged heating significantly reduced the viability of cells included in NIRBMP-2-HG, cells still retained the capacity to produce significant amounts of transgenic growth factor. An irradiation time of $10 \mathrm{~min}$, which induces the production of substantial amounts of BMP-2 without affecting cell viability severely, was selected as optimal and used for all subsequent experiments. To study the degradation profile of NIR-BMP-2-HG, hydrogels were subjected or not to two rounds of NIR irradiation in the presence of rapamycin and cultured up to 45 days after polymerization. A progressive shrinkage of NIR-BMP-2-HG hydrogels, irradiated or not, was detected along the observation period (Fig. S3). NIR irradiation of hydrogels slowed their degradation, as evidenced at 30 and 45 days by the significant higher diameter of irradiated hydrogels. 
Next, NIR-BMP-2-HG were exposed to NIR irradiation for $10 \mathrm{~min}$ in the presence of $100 \mathrm{nM}$ AP21967, a chemically modified derivative of rapamycin that can trigger rapamycin-activated gene switches and is greater than 100 -fold less suppressive of mTOR activity [42] This concentration of dimerizer resulted in maximal induction of transgene expression driven by a heat-activated and rapamycin-dependent system in $\mathrm{C} 3 \mathrm{H} / 10 \mathrm{~T} 1 / 2$ cells subjected to thermal challenge in a thermostatic waterbath [29]. One day after first NIR irradiation, BMP-2 release to the conditioned media of AP21967treated hydrogels was similar to that achieved using $10 \mathrm{nM}$ rapamycin, increasing about 16 times the BMP-2 secretion shown by non-irradiated constructs (Fig. 1E). A second round of NIR irradiation in the presence of AP21967, applied five days after the first round, re-induced the production of BMP-2 in NIR-BMP-2-HG to levels similar to those achieved after first activation.

\subsection{Bioactivity of BMP-2 secreted from NIR-responsive constructs}

To assess whether BMP-2 secreted by induced NIR-BMP-2-HG was bioactive, its autocrine and paracrine effects were investigated. To analyze autocrine bioactivity, we compared the transcriptome of cells residing in constructs that were subjected to two rounds of NIR-irradiation in the presence of dimerizer, $10 \mathrm{nM}$ rapamycin or $100 \mathrm{nM}$ AP21967, with the transcriptome of cells that were treated with the corresponding dimerizer but not NIR-irradiated (Fig. 2A). To narrow our study to genes regulated by secreted BMP-2, excluding those that could be modulated by effects driven by the specific dimerizer, the analysis was restricted to protein-coding genes whose transcript levels significantly increased or decreased $(\mathrm{p}<0.05)$ by at least 1.5 -fold in NIRirradiated NIR-BMP-2-HG in the presence of rapamycin as well as in the presence of rapalog. The analysis identified 160 genes positively regulated and 13 genes negatively regulated in BMP-2 overexpressing cells (Table S2). Results obtained for the 50 most 
highly induced genes can be visualized in the two first columns of the left heatmap in Figure 2B. Interestingly, the gene ontology analysis revealed a range of highly enriched biological processes related to antiviral activity, including “defense response to virus”, “cellular response to interferon-alpha”, “cellular response to interferon-beta” and “response to interferon-gamma” (Table S3 and Figure 2C). Various other processes were also enriched including, most notably, “cell division” and "cell cycle”. The levels of transcripts regulated in BMP-2 overexpressing cells were comparatively investigated in NIR-BMP2-HG that, in the absence of dimerizer, were subjected to two rounds of NIR-irradiation or non-irradiated. Only 9 (5.6 \%) of the 160 genes upregulated by irradiation in the presence of dimerizers, were significantly induced by NIR irradiation alone (Table S2; see also the third column of left heatmap of Figure 2B ). In the absence of dimerizers, NIR-irradiation significantly increased the transcript levels of 149 genes, while 115 were repressed (Table S4). Results obtained for the 50 most highly NIRinduced and repressed genes can be visualized in the two first columns of the middle and left heatmap in Figure 2B. Gene ontology analysis of these sets of genes did not reveal any enrichment of the biological processes found in BMP-2 overexpressing cells (Table S3). The pattern of NIR-regulated genes was remarkably different when irradiation was conducted in the presence of rapamycin or AP21967, as NIR only stimulated the expression of 11 (7.4\%) or 50 (33.6\%), respectively, of the 149 genes induced by NIR alone (Table S4; two first columns of middle heatmap of Figure 2B). Differences were even more pronounced when comparing the expression levels of NIRrepressed genes. Irradiation conducted in the presence of rapamycin or AP21967 only down-regulated the expression of 3 (2.6\%) or 14 (12.2\%), respectively, of the 115 genes that were found repressed by NIR alone (Table S4; two first columns of right heatmap of Figure 2B). 
The paracrine bioactivity of BMP-2 secreted by induced NIR-BMP-2-HG was assayed employing a high density micromass culture of multipotent C3H/10T1/2 stem cells isolated from mouse embryos [43] (Fig. 3A). As reported elsewhere, chondrogenesis differentiation of C3H/10T1/2 cells is induced by BMP-2, which plays a central role in the development of bone and cartilage, and requires cell-cell interaction [44]. $\mathrm{C} 3 \mathrm{H} / 10 \mathrm{~T} 1 / 2$ micromasses treated with 1-100 ng $\mathrm{mL}^{-1}$ rBMP-2 exhibited an extracellular matrix that stained with alcian blue. Staining intensity increased steadily with increasing doses of the recombinant growth factor (Fig. 3B). Mammalian target of rapamycin (mTOR) signaling controls osteoblast proliferation as well as the early stage of osteoblast differentiation. Rapamycin, a specific inhibitor of mTOR, significantly inhibits in vitro proliferation and differentiation of preosteoblastic cells and primary mouse bone marrow stromal cells at concentrations as low as $0.1 \mathrm{nM}$ [45]. In order to evaluate the possible interference by the dose of rapamycin used to conditionally induce NIR-BMP-2-HG on the cell response to BMP-2, C3H/10T1/2 micromass cultures were exposed to rBMP-2 in the presence of $10 \mathrm{nM}$ rapamycin (Fig. 3B). In response to the recombinant growth factor, micromasses exposed to the mTOR inhibitor showed lower levels of alcian blue staining than those differentiated in the absence of the dimerizer. Moreover, the staining levels of cell cultures treated with $1 \mathrm{ng} \mathrm{mL}^{-1} \mathrm{rBMP}-2$ in the presence of rapamycin were not statistically different from the untreated group, indicating that the dimerizer negatively influences the chondrogenic differentiation of C3H/10T1/2 cells induced by rBMP-2 treatment. In a parallel set of experiments, rapamycin was replaced with an analogous concentration of rapalog AP21967. In response to rBMP-2, C3H/10T1/2 micromasses treated with AP21967 showed similar levels of alcian blue staining to cell cultures that only received the growth factor even when a 10-fold increase of rapalog concentration was used (Fig. 3B). The bioactivity of 
induced BMP-2 was assessed using a transwell culture system in which C3H/10T1/2 micromasses were co-cultured with NIR-BMP-2-HG that had been irradiated with NIR light in the presence of $100 \mathrm{nM}$ AP21967 (Fig. 3C). Intensities of alcian blue staining of micromasses co-cultured with induced NIR-BMP-2HG were significantly higher than those exposed to $10 \mathrm{ng} \mathrm{mL}^{-1}$ rBMP-2 and $100 \mathrm{nM}$ AP21967 (Fig. 3D). Alcian blue staining of micromasses cultured with hydrogels that were only treated with AP21967 or only NIR-irradiated was equivalent to micromasses treated with rapalog alone.

\subsection{Induction of transgenic expression in bone lesions}

To evaluate the in vivo activity of heat-activated and ligand-dependent gene switches in NIR-responsive constructs implanted in injured bone, we created $4 \mathrm{~mm}$ diameter calvarial defects in the left parietal bones of mice. The osteotomy was performed using a microsurgery approach that employs a dental drill coupled to a micromotor, preventing damage in the dura mater and minimizing erosion of the periosteum that surrounded the bone lesion. C3H/10T1/2-fLuc cells, which express fLuc under the control of the heat-activated and rapamycin-dependent gene switch [29], were injected together with the precursors of NIR-responsive hydrogels to fill the bone defect. After one and eight days, rapamycin was administered intraperitoneally and 90 min later the implantation area was irradiated for 10 min with an NIR laser. To minimize the risk of skin burns, the laser was adjusted to a delivery power of $11-17 \mathrm{~mW} \mathrm{~mm}^{-2}$ which allowed to increase the temperature of the skin from $34.53 \pm 0.74{ }^{\circ} \mathrm{C}$ to a maximum of $46{ }^{\circ} \mathrm{C}$ on the irradiated surface (Fig. $\mathbf{4 B}$, left panel). The irradiation rounds performed one and eight days after hydrogel injection led to increases of $12.38 \pm 0.62{ }^{\circ} \mathrm{C}$ and 12.17 $\pm 0.73^{\circ} \mathrm{C}$, respectively, in the irradiated surface of mice (Fig. $4 \mathbf{B}$, right graph). One day after illumination with the NIR laser, bioluminiscence imaging revealed strong induction of fLuc activity in the implantation areas (Fig. 4C, left panel). There were no 
significant differences between the levels of fLuc activity induced after the first and second round of NIR irradiation (Fig. 4C, right histogram). Negligible activity levels of the reporter transgene were detected in non-irradiated implants from animals treated only with rapamycin.

To test whether NIR-responsive hydrogels can induce the production of BMP-2 in bone lesions, NIR-BMP-2-HG were polymerized in situ to fill in critical-size defects of calvarial bone. One and eight days after hydrogel injection, implanted animals were treated with rapamycin and exposed to NIR laser emitting at $11-17 \mathrm{~mW} \mathrm{~mm}^{-2}$, which increased the temperature of the skin covering the implant by $12.36 \pm 0.32{ }^{\circ} \mathrm{C}$ and 12.32 $\pm 0.37^{\circ} \mathrm{C}$, respectively (Fig. 4D). One day after NIR irradiation, implants were retrieved and used to prepare extracts. BMP-2 was effectively induced in samples from illuminated implants, although the first round of activation exceeded the second in the total levels of produced growth factor (Fig. 4E).

\subsection{Bone regeneration induced by activated NIR-BMP-2-HG}

In other set of experiments, rapamycin-treated animals that had been implanted with NIR-BMP-2-HG were subjected to two NIR-irradiation rounds as described above and, 10 weeks after implantation, analyzed using X-ray imaging (Fig. 5B, left). Radiographs of non-irradiated animals revealed open bone defects with an average diameter of $4 \mathrm{~mm}$

(Fig. 5B, right). Interestingly, NIR-irradiated animals presented regions of X-ray attenuation inside the calvarial defect which resulted in the reduction of $1 \mathrm{~mm}$ in the radiolucent area diameter that comprised the osteotomy. In non-irradiated mice, implantation areas where osteotomy edges were $3 \mathrm{~mm}$ apart showed the persistence of fibrous tissue bridging the defect, which was principally composed of collagen that stained with aniline blue after Masson's trichrome staining (Fig. 5C, right). At same 
site, the bone defects that had been NIR-irradiated showed a solid bridge of soft tissue progressing to ossification as revealed by the appearance of areas of mineralized collagen. In these animals, the panniculus adiposus in the hypodermis that covers the bone defect area was highly enlarged. Histomorphometric analysis of histological sections confirmed a marked increase in areas of new bone formation within calvarial defects of animals treated with NIR laser (Fig. 5C, right). It should be noted that 10 weeks after implantation, none of animals, NIR-irradiated or not, retained traces of the implanted hydrogels. To test whether NIR-irradiation alone was enough to enhance bone healing, hydrogels that polymerized with or without HGNP, in the absence of C3H-BMP-2 ${ }^{\text {high }}$ cells, were implanted in the defects created in calvarial bone. One and eight days after injection of hydrogels, implanted animals were exposed to NIR laser as above described. During the first and second round of irradiation, animals implanted with hydrogels devoid of HGNP increased the temperature of the skin covering the implant by $4.95 \pm 0.15^{\circ} \mathrm{C}$ and $4.33 \pm 1.08^{\circ} \mathrm{C}$, respectively, likely due to NIR scattering and absorption of the incident light passing through hair follicles, skin and the fibrin matrix Thermal increases elicited by irradiation of implants including HGNP were similar to the above-described experiments including C3H-BMP-2 ${ }^{\text {high }}$ cells. Histological analysis performed 10 weeks after implantation only showed fibrous tissue bridging the defects of irradiated and not irradiated mice, without any sign of bone healing (Fig S4). No differences were observed between mice implanted with hydrogels containing or not HGNP.

\section{Discussion}

Achievement of temporal and spatial control of gene expression remains an important goal in the gene therapy field. Such control would potentially improve safety and efficacy of regenerative factor production. Timely administration of viral vectors 
engineered to constitutively express BMP-2 has been explored as an attempt to define temporal patterns in the production of the osteogenic factor. Thus, injection of adenovirus encoding a constitutively expressed BMP-2 five to ten days after creating a critical-size rat femur defect improved new bone formation when compared with animals receiving the virus at earlier times [20, 21]. Although diminished inflammation and formation of a stable hematoma at delayed times may favour vector access to target cells, it cannot be excluded that fitting the peak activity of transgenic BMP-2 to endogenous wound-healing responses contributed critically to the success of this approach. More sophisticated strategies for adjusting the timing of BMP-2 expression are based on inducible expression systems, designed to overcome the limitations of constitutive promoters. Tetracycline-dependent systems, which rely on transactivators regulated by tetracycline or its analog doxycycline, are examples of inducible systems used for temporal control of BMP-2 expression. Transplantation of genetically engineered stem cells for expressing BMP-2 under the control of tetracycline-dependent systems promoted the healing of nonunion radius fractures [46] and critical-size calvarial defects in mice [47]. However, excessive bone formation was observed in some samples, which was attributed to the expression of BMP-2 beyond the desired temporal window after discontinuation of doxycycline. This phenomenon may be linked to the bone tropism of tetracycline and its derivatives, which interfere with regulated expression due to their accumulation in mineralized tissue [48]. On the other hand, leakiness of tetracycline-dependent systems, which arises from the affinity that some cellular factors have for their transactivator-responsive promoters, results in the expression of significant amount of the targeted transgene in the uninduced state thus compromising the stringency of the inducible system [49]. To overcome these problems, our previous research explored dimerizer-based gene switches to control the 
temporal pattern of BMP-2 expression. This technology is based on the use of a heterodimeric transcription factor composed of separate activation and DNA-binding domains that only form a functional transactivator in the presence of a small dimerizer molecule such as rapamycin or rapalogs that retain dimerizing activity [50]. We engineered a dimerizer-based gene switch to control BMP-2 expression that tightly regulated the in vivo production of the growth factor [38]. Repeated rapamycin treatment over several weeks led to uniform bone formation without bone overgrowth in a calvarial bone defect filled with a collagen hydrogel entrapping fibroblasts that stably harboured the gene switch in their genome. Conversely, cells transduced with an adenovirus carrying a constitutively expressed BMP-2 formed highly irregular bone that was discontinuous with the surrounding tissue. These findings showed that sustained low levels of BMP-2 provided by the inducible system improved the regenerative outcome when compared with the high, but transient, levels of transgene activity driven by the constitutive system. In the present study we assessed the impact on bone healing of BMP-2 expression controlled by a rapamycin-dependent gene switch that is activated by photothermal heating thereby providing temporal and spatial control of transgene production. We previously reported that the optical properties of fibrin-based hydrogels incorporating HGNP can be efficiently exploited to deliberately control in vivo the spatiotemporal production of $\mathrm{hVEG}_{165}$ by means of a heat-activated and rapamycindependent gene switch [32]. Data herein show that same approach can be used to control the secretion of BMP-2 in bone defects. First, we determined in vitro the optimal HGNP concentration and time of NIR irradiation to induce BMP-2 expression in genetically engineered cells entrapped within the hydrogels. Parameters for the generation of sustained mild hyperthermia were selected to maximize the levels of growth factor production from transgenic cells while minimizing the risk of heating 
damage. As compared to in vitro activation, generation of mild hyperthermia in implanted NIR-responsive hydrogels requires downward adjustment of deposited NIR energy. Thus, NIR irradiation for $10 \mathrm{~min}$ at $11-17 \mathrm{~mW} \mathrm{~mm}^{-2}$ after rapamycin administration led to robust transgene expression in subcutaneously implanted NIRresponsive constructs that incorporated cells harbouring the heat-activated and rapamycin-dependent gene switch [32]. As shown in the present work, similar activation conditions resulted in the induction of fLuc activity which matched the NIR irradiation spot in implants that filled the bone defects. Induced transgene expression was detected in implanted hydrogels not only after a first round of activation but also after a second round performed one week later. This methodology, i.e. two rounds of rapamycin administration followed by NIR illumination of photothermal cell constructs, increased transgenic BMP-2 production in osteotomized regions and led to the formation of new mineralized tissue thereby reducing the size of the treated bone lesions. Moreover, the use of a dimerizer-dependent transactivator that is expressed upon heat activation not only prevented incidental transgene activation triggered by hyperthermia associated with post-traumatic immune responses, but also allowed us to deliberately emulate the timing of sequential BMP-2 production seen during normal bone healing where BMP-2 expression is first detected during early inflammation and subsequently increases during the periosteal response [51].

One possible concern with using the heat-activated and rapamycin-dependent gene switch for bone healing relates to the deleterious effect of immunosuppressive drugs as rapamycin on bone metabolism and development [52-55]. In particular, a preclinical study in mice concluded that rapamycin can delay fracture healing, most probably by inhibiting cell proliferation and neovascularization in the callus [56]. Our bioactivity studies showed that rapamycin concentration used to conditionally express BMP-2 
reduced the chondrogenic differentiation of $\mathrm{C} 3 \mathrm{H} / 10 \mathrm{~T} 1 / 2$ micromass cultures, interference that was not observed when rapalog AP21967 was employed as dimerizer. Similar to what is seen for naturally occurring dimerizer molecules, derivatization of rapamycin can dramatically lower the cell permeability of the original compound. Thus, AP21967 was observed to have $1 / 25^{\text {th }}$ the activity of rapamycin when its dimerization activity was measured in vitro [57]. Also, this rapalog has short half-life in mouse plasma $(<4 \mathrm{~h})$ [58]. In view of these considerations we chose to use rapamycin as dimerizer for in vivo studies. Future work will explore whether using nonimmunosuppressive AP21967 instead of rapamycin may be advantageous to enhance the regenerative capacity of conditionally expressed BMP-2, e.g. augmenting or accelerating new mineralized tissue formation in the created bone lesion.

Previous in vitro reports indicate that mild heat may stimulate the proliferation and/or differentiation of osteoblast precursors, thereby promoting bone regeneration [59, 60]. However, our comparative transcriptome studies of cells residing in NIRBMP-2-HG that, in the absence of dimerizer, were NIR-irradiated or non-irradiated indicated that the thermal stimuli employed by us do not affect the expression level of genes related to proliferation or to the acquisition of a differentiated phenotype. Similar comparative analyses performed in cells entrapped in hydrogels that were NIRirradiated or no-irradiated in the presence of dimerizers revealed a potent autocrine effect of the growth factor in BMP-2 overexpressing cells, as demonstrated by the induction of genes related to cell cycle. In agreement with publications reporting the antiviral role of BMPs [61, 62], the autocrine response to BMP-2 was characterized by a signature of induced genes related to virus-induced and interferon associated responses. Some recent publications reported that magnetic- and NIR-responsive implants may enhance in vivo bone regeneration through thermal stimulation [63, 64]. However, we 
have not evidenced any bone formation in NIR-irradiated defects implanted with plasmonic hydrogels devoid of cells, which highlights the essential role of secreted BMP-2 on the healing effects observed in NIR-irradiated and rapamycin-treated NIRBMP-2-HG. Future work will determine whether NIR-responsive substrates other than the fibrin-based hydrogels used in the present work, may improve BMP-2-driven bone healing through a thermal effect.

In summary, we have shown that deliberate patterning of transgene expression in NIRresponsive hydrogels implanted in a bone defect may be a useful approach for achieving spatiotemporal control of BMP-2, thereby mimicking endogenous tissue repair mechanisms. The developed platform, which is not restricted to specific photothermal nanomaterials or scaffold composition, can be refined for in vivo gene therapy approaches that employ viral vectors encoding the heat-activated and rapamycindependent gene switch. In this approach, the NIR-responsive hydrogel could serve as a reservoir of these vectors to deliver the gene switch to target cells while providing a source of local hyperthermia to enable transgenic induction. Inadvertent activation of transgene expression from virus that might disseminate from the NIR-responsive hydrogels and gain access to major organs can be excluded, as the gene switch cannot be activated in the absence of dimerizer administration and heat activation. While the current study provides proof of concept for the application of NIR laser irradiation as a means of inducing safe and timed transgene expression aimed at regenerating lost bone, further optimization of the technological approach is needed to achieve the formation of fully functional new bone.

\section{Conclusions}


We explored the bone regenerative potential of a therapeutic platform based on the combination of inducible transgene expression and the technology of NIR-responsive hydrogels. Cells harbouring a gene expression system triggered by heat and dependent on a low molecular weight ligand to control BMP-2 expression were entrapped in scaffolds based on fibrin and plasmonic gold nanoparticles. After ligand administration, BMP-2 production was induced in vitro in cell constructs that efficiently transduced incident NIR energy into mild heat. BMP-2 produced in induced cell constructs promoted the chondrogenic differentiation of three-dimensional cultures of multipotent stem cells, indicating that the transgenic growth factor was bioactive. NIR-responsive cell constructs were injected to fill a critical-size defect of calvarial bone created in mice. NIR illumination of implants in animals administered with ligand induced BMP-2 production in the bone lesion, which ultimately led to a significant increase in new mineralized tissue. In summary, the platform developed here for the stringent spatiotemporal control of BMP-2 reveals broad potential in the field of bone regenerative medicine.

\section{ACKNOWLEDGEMENTS}

The authors would like to thank Dr. Sara Ballester (Unidad Funcional de Investigacion en Enfermedades Cronicas, Instituto de Salud Carlos III (ISCIII)) for assistance in radiographical examinations and valuable discussions. The authors thank Jesus García and Pedro Botias (Unidad de Genómica-Universidad Complutense de Madrid/Parque Científico de Madrid, Spain) (microarray analyses), as well as Dr Ricardo Ramos and Susana Ovalle (Unidad de Genómica-Universidad Autónoma de Madrid/Parque Científico de Madrid) (Taq-Man gene expression studies) for their excellent technical assistance. This work was supported by grant PI15/01118 from ISCIII-Fondos FEDER, Ministry of Economy and Competitiveness (MINECO), Spain, grant RTI2018-095159- 
B-I00 from Ministerio de Ciencia, Innovación y Universidades (MICINN), grant Roche-IdiPAZ from the intramural funding program of Foundation for Biomedical Research of Hospital Universitario La Paz-IdiPAZ, grant ERC-2013-CoG-614715 (NANOHEDONISM) from ERC Consolidator Grant program, grant R21AR072336 from NIH and by HSF Pharmaceuticals S.A. C.E-D. was the recipient of predoctoral grant FI14/00447 from ISCIII-Fondos FEDER, MINECO. N.V. is supported by Program I2 from CAM.

Declarations of interest: The work described herein was partially supported by contracts from HSF Pharmaceuticals S.A. to N.V. 